\section{ORIGINAL RESEARCH}

\author{
C.H. Yen \\ M.M.H. Teng \\ W.H. Yuan \\ Y.C. Sun \\ C.Y. Chang
}

\title{
Preventive Vertebroplasty for Adjacent Vertebral Bodies: A Good Solution to Reduce Adjacent Vertebral Fracture after Percutaneous Vertebroplasty
}

BACKGROUND AND PURPOSE: Adjacent VCF frequently occurs after percutaneous vertebroplasty. Our aim was to evaluate PrVP in the prevention of PVNO-adjacent VCF.

\begin{abstract}
MATERIALS AND METHODS: Radiographs of 68 patients who initially presented with a single-level unhealed fracture and underwent vertebroplasty were retrospectively reviewed for the occurrence of PVNO fracture. Patients in the nonpreventive group $(n=33)$ underwent TVP only for a vertebra with an unhealed fracture. The preventive group $(n=35)$ underwent PrVP combined with TVP. We injected bone cement into the caudal part of the superior adjacent vertebra and the cephalic part of the inferior adjacent vertebra to perform PrVP.
\end{abstract}

RESULTS: The incidences of PVNO fracture in adjacent vertebra next to a vertebra cemented at the patient's first vertebroplasty (within 6 months: $24 \%$ versus $3 \%, P=.012$; within 1 year: $30 \%$ versus $3 \%, P=.006 ;>4$ years: $39 \%$ versus $3 \%, P=.006$ ) markedly decreased in the preventive group compared with the nonpreventive group. PVNO fracture was found in $26 \%$ of vertebrae adjacent to the first TVP level in the nonpreventive group and in $2 \%$ of vertebrae adjacent to a PrVP level in the preventive group after inclusion of all PrVP procedures. Approximately $33 \%$ of patients in the nonpreventive group underwent repeat vertebroplasty, mainly due to adjacent fractures. Only $3 \%$ of patients in the preventive group underwent repeated procedures. None of the vertebrae cemented for PrVP or TVP developed PVNO refracture.

CoNCLUSIONS: Preventive vertebroplasty for the adjacent vertebra combined with TVP for the fractured vertebra is effective in the prevention of propagation of PVNO adjacent fractures, thus reducing the necessity of multiple repeat vertebroplasty procedures.

ABBREVIATIONS: PrVP = preventive vertebroplasty; PVNO = postvertebroplasty new-onset; TVP $=$ therapeutic vertebroplasty; $V C F=$ vertebral compression fracture

$\mathbf{P}$ ercutaneous vertebroplasty has become a therapeutic option for the management of painful tumor or osteoporosis-related VCF with poor response to medical treatment. Percutaneous vertebroplasty is a minimally invasive procedure that involves filling fractured vertebrae with bone cement to relieve pain. It has been shown to facilitate substantial pain relief and aid in rehabilitation in up to $90 \%$ of patients as early as $1-2$ days after the intervention. ${ }^{1-5}$

The overall complication rate associated with percutaneous vertebroplasty is reported to be $1 \% \sim 3 \%$. However, some patients experience new VCFs soon after the procedure. ${ }^{1-12}$ This complication often occurs on the adjacent

Received June 29, 2011; accepted after revision September 5.

From the Department of Radiology (C.H.Y., M.M.H.T., W.H.Y., Y.C.S., C.Y.C.), Taipei Veterans General Hospital, Taipei, Taiwan, R.O.C.; Department of Biomedical Imaging and Radiological Sciences (C.H.Y., M.M.H.T.) and School of Medicine (M.M.H.T., W.H.Y., C.Y.C.), National Yang-Ming University, Taipei, Taiwan, R.O.C.; and Department of Radiology (C.H.Y.), Taipei Hospital, Department of Health, Taiwan, R.O.C.

This work was partially supported by the National Science Council of R.O.C. (grant No. NSC 95-2314-B-010-001) and Taipei Veterans General Hospital Research Program (grant No. V100C-158).

Please address correspondence to Michael Mu Huo Teng, MD, Department of Radiology, Taipei Veterans General Hospital, 201 Sect 2, Shih-Pai Rd, Peitou, Taipei, 11217 Taiwan; e-mail: mhteng@gmail.com

Indicates open access to non-subscribers at www.ajnr.org

http://dx.doi.org/10.3174/ajnr.A2898 vertebrae in approximately half of the cases, especially in the inferior endplate immediately superior to the treated level or the superior endplate of the vertebrae immediately inferior to the treated vertebrae. ${ }^{1}$ The object of this study was to evaluate the efficacy of PrVP in the prevention of PVNO VCF in the adjacent vertebral bodies (adjacent fractures).

\section{Materials and Methods}

Radiographs of 247 patients with VCFs who had undergone percutaneous vertebroplasty from June 2000 to August 2006 in our hospital were retrospectively reviewed for the occurrence of PVNO VCF. We enrolled 68 patients who initially presented with a single level of unhealed VCF, had undergone percutaneous vertebroplasty, and had received follow-up plain films of the thoracic and lumbar spine after vertebroplasty. All patients enrolled had osteoporosis. Patients with bone tumor were excluded. The study included 33 patients in the nonpreventive group who underwent TVP at the level of the unhealed fracture from June 2000 to April 2005 ( 16 men, 17 women; mean age, 77.3 years; age range, $62-89$ years) and 35 patients in the preventive group who underwent TVP and PrVP for normal-shaped adjacent vertebrae next to the unhealed fracture from January 2004 to August 2006 ( 15 men, 20 women; mean age, 77.2 years; age range, 64-90 years). The adjacent vertebral body was excluded from PrVP if it was S1 or if it had a healed old fracture.

The distribution of the initial vertebral fractures is listed in Table 


\begin{tabular}{lccccccccccccc}
\hline Table 1: The locations of the initial vertebral body fractures \\
\hline & T6 & T7 & T8 & T9 & T10 & T11 & T12 & L1 & L2 & L3 & L4 & L5 & Total \\
\hline Nonpreventive group & 1 & 1 & 0 & 0 & 0 & 0 & 14 & 7 & 4 & 5 & 1 & 0 & 33 \\
Preventive group & 0 & 1 & 0 & 0 & 2 & 1 & 9 & 13 & 6 & 3 & 0 & 0 & 35 \\
\hline
\end{tabular}

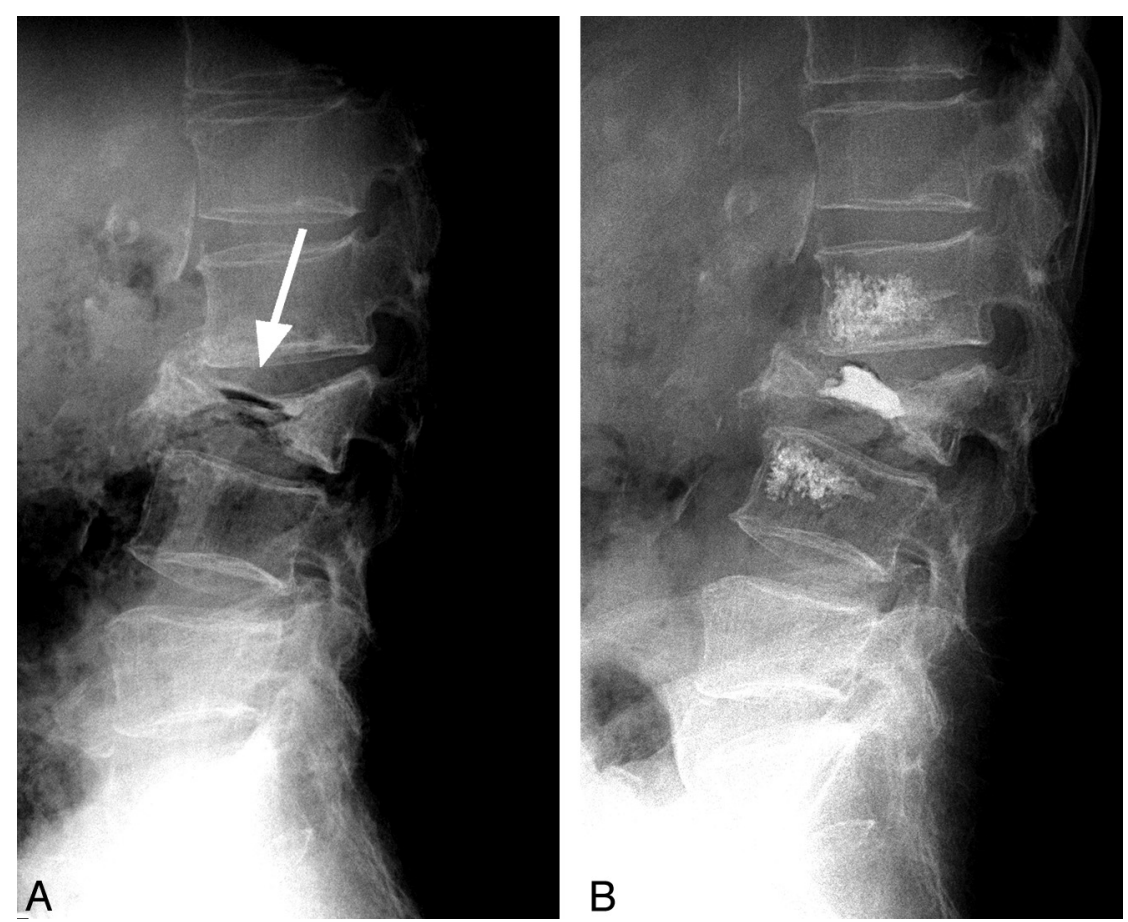

Fig 1. An example of a PrVP. A 69-year-old man with severe back pain. $A$, Grade 3 vertebral body fracture with a gas-containing cavity (arrow) inside was found at $\mathrm{L} 3$ in a plain film obtained 1 month before vertebroplasty. $B$, He underwent TVP for the L3 vertebral body and preventive cementing for the lower L2 and upper L4. Image was obtained 8 months after vertebroplasty. In this case, L1 and L5 were adjacent vertebral bodies next to a PrVP level for evaluation.

1. All patients gave written informed consent after a thorough explanation of the percutaneous vertebroplasty procedure, including preventive treatment for the adjacent vertebral bodies. The mean length of follow-up was $940 \pm 826$ days (ranging from 7 to 2495 days) in the nonpreventive treatment group and $482 \pm 503$ days (ranging from 7 to 1885 days) in the preventive treatment group.

Preoperative imaging assessment included spine plain film and MR imaging (sagittal T1-weighted, sagittal T2-weighted, sagittal short $\tau$ inversion recovery, axial T1-weighted, and axial T2-weighted images). All patients had painful unhealed VCFs with MR imaging evidence of edema and enhancement or the presence of avascular necrosis inside the vertebral body with fracture. Vertebroplasty was not considered in patients with associated posterior column fracture or severe spinal stenosis. Follow-up plain films after vertebroplasty included routine anteroposterior and lateral films of the thoracic and lumbar spine without weight-bearing.

Percutaneous vertebroplasty was achieved by using a unilateral transpedicular approach with an 11-gauge bone biopsy needle advanced to the anterior third of the vertebral body under fluoroscopic guidance. The bipedicular approach was adopted only when the cement could not reach the midline of the vertebral body. Vertebroplastic cement was mixed following manufacturer recommendations. The cement was injected by using 3- or 5-mL syringes manually. The amount of bone cement injected at TVP was adjusted for each vertebral body according to the presence or absence of avascular necrosis and the size of the cavity of avascular necrosis. When there was a cavity inside the vertebral body, we tried to fill the cavity as much as possible. We tried to leave the posterior one-third of the vertebral body uncemented. The amount of bone cement injected in 1 vertebra during TVP was approximately $3-5 \mathrm{~mL}$ without avascular necrosis, $4-6 \mathrm{~mL}$ with a small cavity inside, and $5-8 \mathrm{~mL}$ with a large cavity inside.

In PrVP, we injected bone cement into the adjacent part of the adjacent vertebral bodies, including the caudal part of the superior adjacent vertebral body and cephalic part of the inferior adjacent vertebral body (Fig 1); other parts of the adjacent vertebral bodies were not cemented. Also, we avoided dense packing of bone cement when performing PrVP. The bone cement volume injected into the adjacent vertebral bodies during PrVP was approximately $2-5 \mathrm{~mL}$. If the cavity, fissure, or fracture in the vertebral body was close to or at the superior endplate, we might have omitted the preventive cementing in the inferior adjacent vertebra. Likewise, if the cavity, fissure, or fracture was close to or at the inferior endplate, we might have omitted the preventive procedure in the superior adjacent vertebra. We performed PrVP for both the superior and inferior adjacent vertebral bodies if the cavity of avascular necrosis was big, both the superior and inferior endplates were thin, or both the superior and inferior endplates had fractures.

We evaluated the fate of the uncemented normal adjacent vertebral bodies and recorded any PVNO adjacent fractures in the follow-up periods, as well as their location above or below the cemented vertebra. The adjacent vertebral bodies included for evaluation had to be normal in shape, with no fracture at the time of vertebroplasty. We evaluated 2 groups of uncemented adjacent vertebral bodies: those 


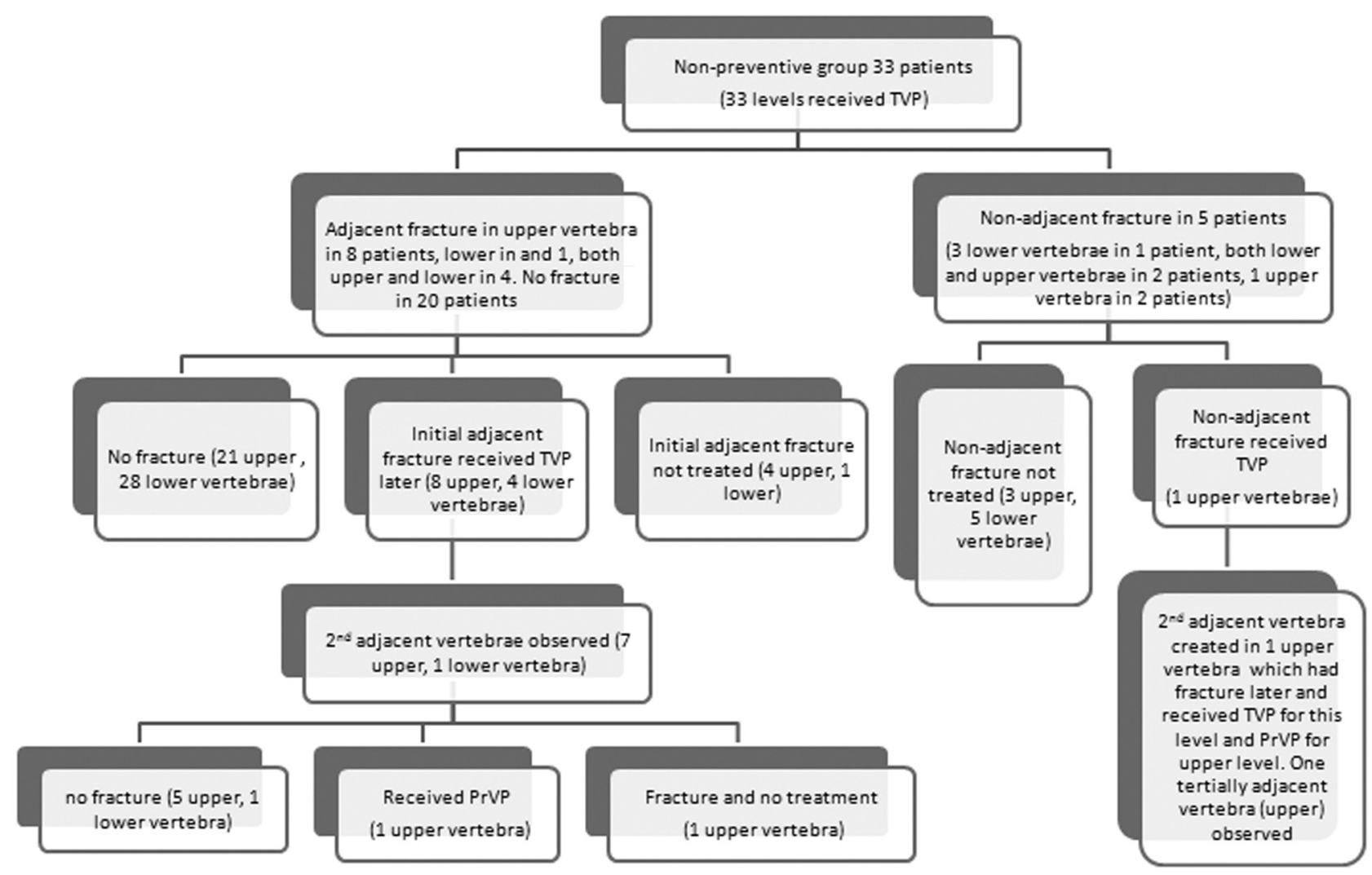

Fig 2. Nonpreventive group.

next to a vertebra cemented for fracture at TVP (next to a TVP level) and those next to a vertebra cemented for prevention at PrVP (next to a PrVP level). In Fig 1, L3 received TVP, and L2 and L4 received PrVP; thus, L1 and L5 were evaluated for adjacent fracture next to a PrVP level.

We studied the cumulative PVNO VCF rate of the initial adjacent vertebral bodies created at the first vertebroplasty within the $<6$ month, $<1$-year, and $>4$-year follow-up periods in both the nonpreventive and preventive treatment groups. The cumulative adjacent fracture rates in the $<6$-month follow-up period in both groups was statistically analyzed by using the Fisher exact test, and the $\chi^{2}$ test with a Yates correction was used for the PVNO adjacent VCF rate at the $<1$ - and $>$ 4-year follow-up periods. $P$ values $<.05$ were considered statistically significant.

Many of these patients underwent multiple vertebroplasty procedures because of painful PVNO VCF, thereby creating more adjacent vertebral bodies for evaluation. We also evaluated the fracture rate of all these adjacent vertebral bodies created at the first and subsequent vertebroplasties.

\section{Results}

Data on the nonpreventive group are presented in Fig 2. Thirteen $(39 \%)$ patients in the nonpreventive group $(n=33)$ developed PVNO VCF in the initial vertebrae adjacent to the first TVP level. Fracture occurred in 17 (26\%) of these initial vertebrae adjacent to the first TVP levels: Twelve (71\%) adjacent fractures occurred in the upper vertebra and 5 (29\%) in the lower vertebra. The incidence of adjacent fracture in the upper vertebra was $36 \%$, and in the lower vertebra, $15 \%$.

Data on the preventive group are presented in Fig 3. PrVP was performed for 53 initial adjacent vertebrae, leaving 17 adjacent vertebrae untreated. One $(2.8 \%)$ of the 35 patients in the preventive group developed PVNO VCF, which occurred next to the TVP level. No adjacent fracture was found in the secondary vertebrae next to the first PrVP level. Adjacent fracture next to a PrVP level was found in only 1 patient who underwent a PrVP combined with a second TVP for a PVNO nonadjacent fracture.

PVNO VCF in adjacent vertebrae was found within 6 months and 1 year following vertebroplasty in 8 (24\%) and 10 (30\%) patients, respectively, in the nonpreventive group. The adjacent fracture in 1 patient in the preventive group was found within 6 months after a TVP. Compared with the nonpreventive group, the occurrence of PVNO VCF in adjacent vertebra next to a vertebra cemented at the patient's first vertebroplasty in the preventive group was markedly decreased, with statistical significance $(P=.012, P=.006, P=.006)$ in these 3 respective follow-up periods ( $<6$ months, $<1$ year, and $>4$ years).

Eleven $(33 \%)$ patients in the nonpreventive group underwent repeat vertebroplasty procedures because of painful PVNO VCF, and $10(91 \%)$ of these repeat procedures were caused by adjacent fracture (Table 2 ). In comparison, only 1 (3\%) patient in the preventive group underwent repeated vertebroplasty procedures. In total, there were 92 adjacent vertebrae (44 superior vertebrae, 48 inferior vertebrae) next to all TVP levels in the first and subsequent vertebroplasty procedures. Twenty (22\%) of these vertebrae adjacent to the TVP levels had fractures involving 15 superior and 5 inferior vertebrae. There were 52 vertebrae (33 superior vertebrae, 19 infe- 


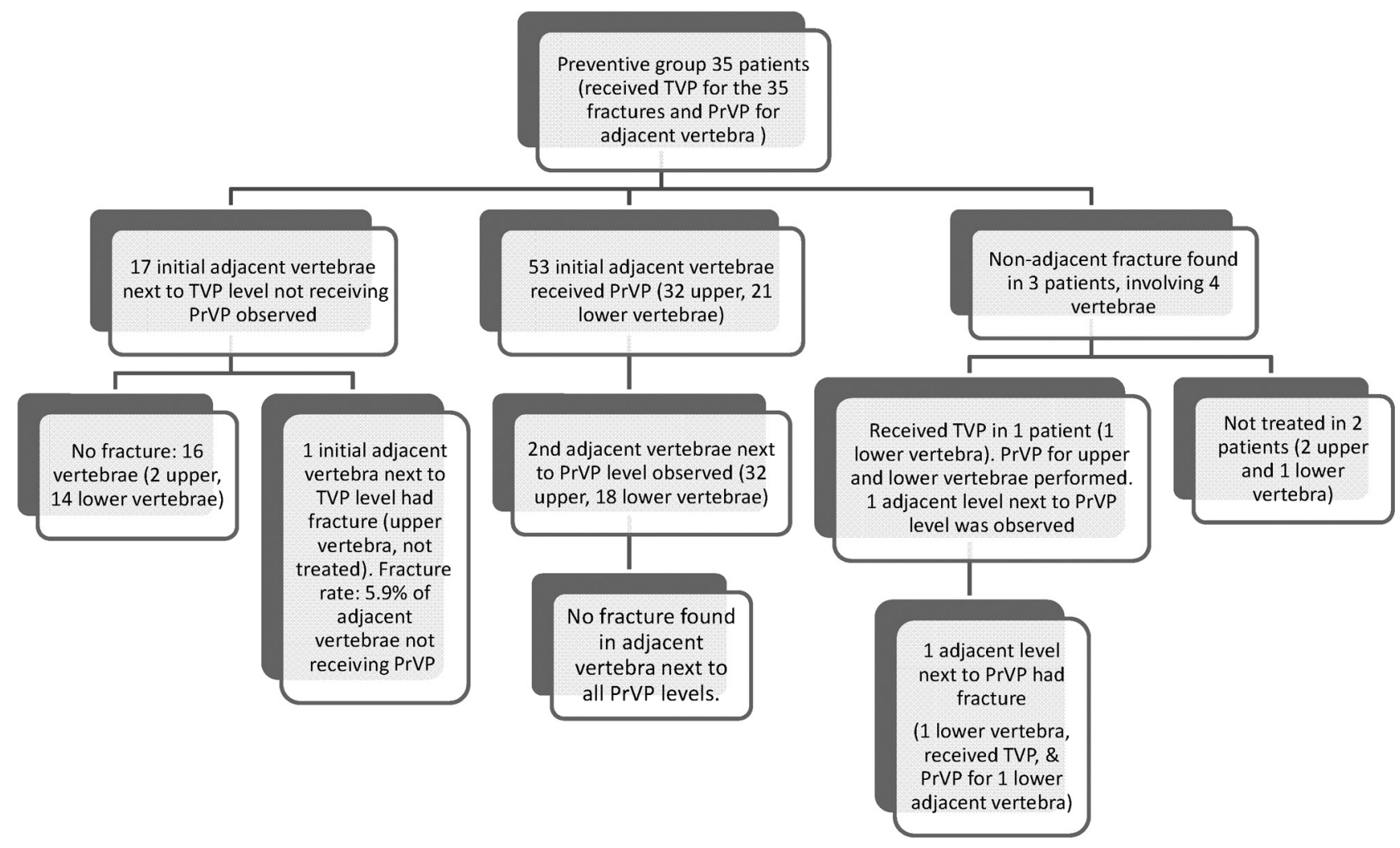

Fig 3. Preventive group.

\begin{tabular}{|c|c|c|c|}
\hline Groups & $\begin{array}{c}\text { No. of } \\
\text { Vertebroplasties }\end{array}$ & $\begin{array}{l}\text { Patient } \\
\text { No. }\end{array}$ & Reason \\
\hline \multirow[t]{3}{*}{$\begin{array}{l}\text { Nonpreventive } \\
\text { group }\end{array}$} & 2 & 9 & $\begin{array}{l}\text { The adjacent vertebral fracture } \\
\text { was in } 1 \text { level only or in } \\
\text { both levels found at the } \\
\text { same time }\end{array}$ \\
\hline & 3 & 1 & $\begin{array}{l}2 \text { adjacent fractures occurred } \\
\text { separately }\end{array}$ \\
\hline & 5 & 1 & $\begin{array}{l}3 \text { adjacent fractures and } 1 \\
\text { nonadjacent fracture } \\
\text { occurred separately }\end{array}$ \\
\hline $\begin{array}{l}\text { Preventive } \\
\text { group }\end{array}$ & 3 & 1 & $\begin{array}{l}\text { Fracture in the vertebra } \\
\text { adjacent to a PrVP level } \\
\text { and } 1 \text { nonadjacent fracture }\end{array}$ \\
\hline
\end{tabular}

rior vertebrae) adjacent to all PrVP levels in the first and subsequent vertebroplasty procedures. Only 1 (2\%) of these 52 vertebral bodies adjacent to the PrVP level had a fracture (1 inferior vertebra).

The survival curve of all adjacent vertebral bodies created in repeat vertebroplasty procedures is shown in Fig 4. An adjacent vertebra without fracture was considered to have survived. The length of survival was from the date of vertebroplasty for the vertebra next to the adjacent vertebral body until the date that the PVNO fracture in the adjacent vertebra was found. The 2-year survival rates of adjacent vertebral bodies without fracture were $97 \%, 90 \%, 78 \%$, and $75 \%$, respectively, for adjacent vertebral bodies next to a PrVP level, all inferior adjacent vertebral bodies, adjacent vertebral bodies next to a TVP level, and all superior adjacent vertebral bodies.

None of vertebral bodies cemented for previously unhealed fracture in TVP and none of vertebrae cemented for prevention in PrVP developed PVNO VCF.

\section{Discussion}

The reported rate of PVNO VCF without PrVP ranges widely, from $0 \%$ to $52 \%$; on average, approximately $20 \%$ occur within 1 year. ${ }^{1}$ According to Lindsay et al, ${ }^{2}$ the PVNO VCF rate within 1 year was $19.2 \%$ among patients with 1 initial VCF and $24 \%$ among patients with a history of $>1$ involved vertebrae. The occurrence of PVNO VCF without PrVP in our study was $30 \%$ within 1 year, which is within the range of previous reports and higher than that in the report of Lindsay et al. ${ }^{2}$

In our study, we evaluated the incidence of fracture in adjacent vertebral bodies. Fracture occurred in $26 \%$ of initial vertebrae adjacent to the first TVP levels in the nonpreventive group. With inclusion of all adjacent vertebrae next to all TVP levels in initial and subsequent vertebroplasty procedures, the incidence was underestimated and became $22 \%$ because of the addition of 17 vertebral bodies less susceptible to fracture. These 17 vertebrae were next to the level that had received TVP, but not PrVP, in the preventive group. They were thought to be less susceptible to developing fracture by the operator because they were the adjacent vertebral body farther from the cavity, fissure, or fracture in the vertebra undergoing TVP. The lower susceptibility to fracture was confirmed by the low incidence of fracture $(5.9 \%)$ in these vertebrae at followup. Therefore, we adopted $26 \%$ as our incidence of adjacent fracture next to the TVP level in the "Discussion" and "Conclusions" sections. Similarly, when we discuss the incidence of adjacent fracture in the upper and lower adjacent vertebrae, we will refer to data of the initial vertebrae adjacent to the first TVP levels in the nonpreventive group. 


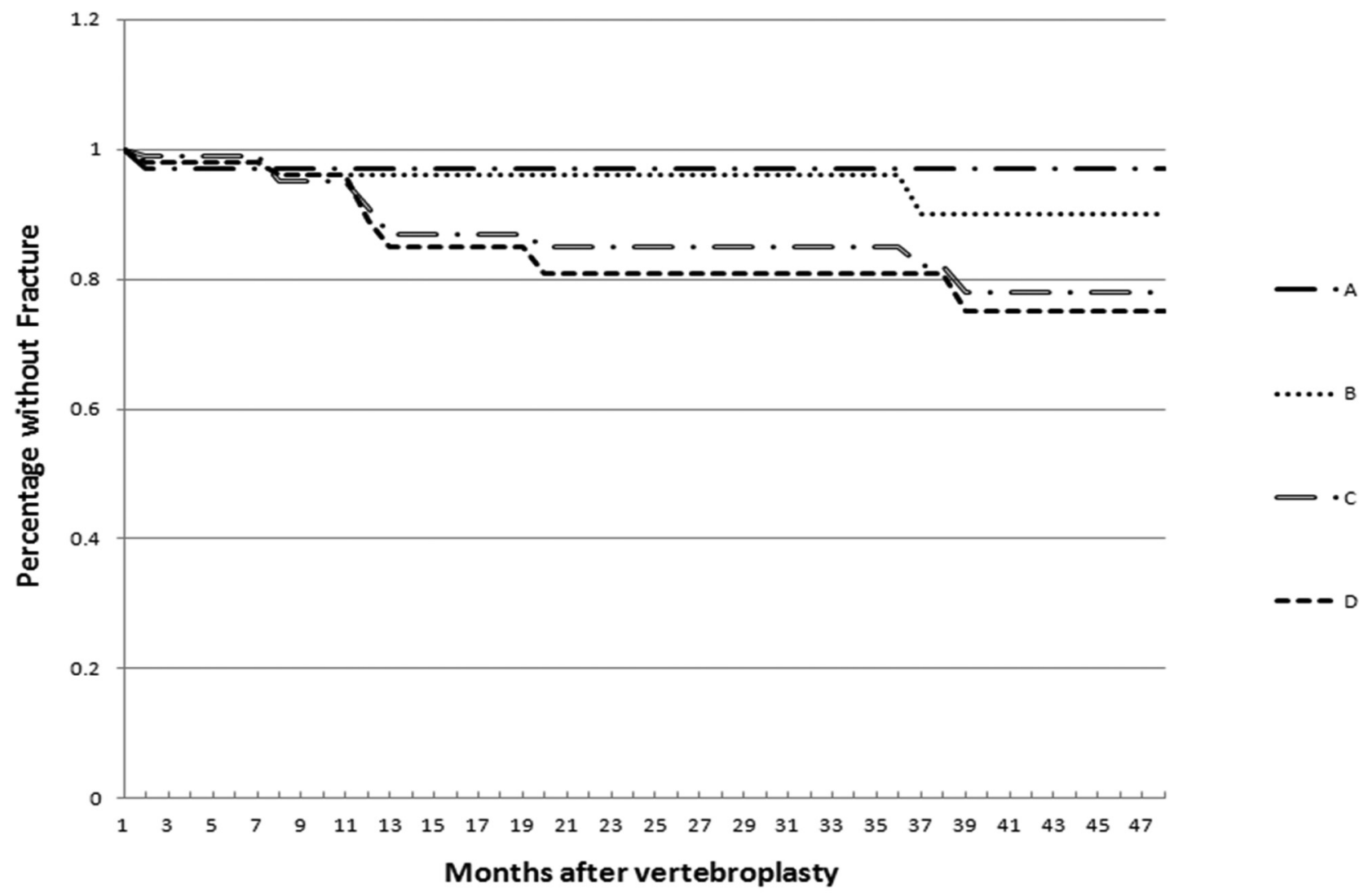

Fig 4. Survival curve of adjacent vertebral bodies. The survival of adjacent vertebral bodies is best for those next to vertebrae cemented for prevention (PrVP) (line A), followed by lower adjacent vertebral bodies (line $B$ ) and vertebral bodies next to vertebrae cemented for previous fracture (TVP) (line C), and worst for upper adjacent vertebral bodies (line $D$ ).

Several studies have suggested that there may be an associated increased risk of fracture in vertebrae adjacent to the percutaneous vertebroplasty augmentation site. ${ }^{1-9}$ This observation has been supported by several ex vivo experiments. ${ }^{8-10}$ In most published vertebroplasty studies, somewhere between $41 \%$ and $67 \%$ of incident fractures occur adjacent to the treated vertebrae. ${ }^{1,7}$ These results suggest that a new adjacent VCF is more likely to occur because of the percutaneous vertebroplasty procedure. Thus, Trout and Kallmes ${ }^{1}$ and Chiang et $\mathrm{al}^{10}$ suggested that PrVP and low-volume vertebroplasty might be appropriate to diminish the risk of new VCF.

Several ex vivo experiments reported the possible biomechanical causes of the higher PVNO VCF rate in adjacent vertebrae and the protective effects of PrVP. ${ }^{8-10}$ Cement in the treated vertebral body "acts like a pillar" that reduces the physiologic inward bulge of the endplates of the cemented level, increases pressure in the disk, and augments inward bowing of the adjacent vertebral endplate causing the adjacent vertebra to be at risk for fracture. ${ }^{1,7}$ VCF in the adjacent vertebrae after percutaneous vertebroplasty is not induced by the elevated stiffness of the treated vertebrae, but instead the anterior shift of the upper body is the dominating factor. ${ }^{13}$ The VCF may be due to the compression flexion induced by the anchoring effect of the facet joint. PrVP strengthens the osteoporotic vertebrae, decreases the progression of vertebral height loss, and hence protects the adjacent intact vertebrae from elevated flexion bending. ${ }^{10}$

PVNO adjacent VCF has been found to be associated with cement extension into the disk space and is correlated with the morphologic extent of bone cement. ${ }^{9,12}$ It was found that only cement leakage into the disk was a significant predictor of new adjacent VCF after percutaneous vertebroplasty. ${ }^{4,5}$ In our nonpreventive group, occurrence of adjacent fracture accounted for almost all fractures at the $<6$-month, $<1$-year, and $>4$-year follow-up periods. As such, PVNO adjacent fracture has been a severe problem in our study, so we designed the PrVP technique of implanting bone cement into the adjacent part of the adjacent vertebrae, leaving the remote part of the adjacent vertebrae uncemented.

In our study, adjacent fracture occurred in $26 \%$ of adjacent vertebrae in the nonpreventive group and in only $2 \%$ of the preventive group. In addition, the 2-year survival rate of adjacent vertebral bodies that had no fracture was best for adjacent vertebral bodies next to the PrVP level (Fig 4). Eleven (33\%) patients in the nonpreventive group underwent repeat vertebroplasty procedures, and most of these were caused by adjacent fracture. In comparison, only 1 (3\%) patient in the preventive group underwent repeat vertebroplasty procedures. Therefore, our strategy to avoid propagation of the adjacent vertebral body fracture by PrVP did work in lowering the incidence of adjacent fracture and reducing the need for multiple repeat vertebroplasty procedures.

Kobayashi at $\mathrm{al}^{3}$ reported a new VCF rate of $22.4 \%$ (20/89) within 1 year after percutaneous vertebroplasty in the nonpreventive treatment group and $9.7 \%(15 / 155)$ within 1 year in the preventive treatment group. In our study, the occurrence of PVNO adjacent VCF within 1 year in the nonpreventive and preventive groups was $30 \%$ and $3 \%$, respectively. The benefit 
of a reduction in new adjacent VCFs within 1 year by using PrVP was 13\% and 27\%, respectively, in our study and that of Kobayashi et al. The reason for the difference in the benefit of PrVP between these 2 studies was probably the difference in the techniques used.

For PrVP, we cemented the adjacent part of the adjacent vertebrae only. The remote part of the adjacent vertebrae remained uncemented to avoid the propagation of fracture to farther adjacent vertebral bodies. Kobayashi et al performed PrVP in only the upper adjacent vertebrae because they thought the incidence of new fractures was relatively low in the lower adjacent vertebrae. Similarly, we found the incidence of new fractures for the upper adjacent vertebrae was higher than that for the lower adjacent vertebra in the initial adjacent vertebrae next to the first TVP levels in the nonpreventive group (36\% versus 15\%). Also, we found $71 \%$ of initial adjacent fractures in the nonpreventive group occurred in the upper vertebrae. Among adjacent vertebral bodies next to the PrVP level, the 2-year survival rate of vertebral bodies without fracture was better for lower adjacent vertebral bodies than for upper vertebral bodies (Fig 4). However, the 29\% initial adjacent fractures in the nonpreventive group that occurred in the lower adjacent vertebral body still could not be ignored.

Becker et $\mathrm{al}^{14}$ reported no difference in the 1-year refracture rate between the monosegmental group $(21.7 \%, 5 / 23)$ and the preventive group $(25.9 \%, 7 / 27)$ by using kyphoplasty. This result differed from ours and that of Kobayashi et al. ${ }^{3}$ This difference could be due to the different preventive modalities used (kyphoplasty versus vertebroplasty). On the other hand, Becker et al limited the PrVP to 1 superior or inferior vertebral body, based on the fracture site.

The limitations of this study include the following: 1) the study was a retrospective, not a randomized trial; 2) nonpreventive treatment was performed first, followed by preventive treatment; and 3) new VCF assessment depended on imaging modalities, but some asymptomatic patients did not want to undergo an imaging study and did not enroll in our study. Some patients in both groups were lost to follow-up early on.

Initially we performed vertebroplasty only for the level with fracture that had avascular necrosis or edema. The period of performing TVP only and TVP combined with PrVP overlapped for approximately 1 year and 4 months. The reason for this overlap was that the concept and necessity of PrVP developed gradually. We developed the PrVP technique because a high frequency $(30 \%)$ of our patients developed PVNO adjacent VCF in the first year of this follow-up study.

The time interval between the first patient undergoing TVP only and the last patient undergoing TVP combined with PrVP was 6 years and 2 months. During this interval, there had been obvious advances in the medical treatment of osteoporosis. For example, raloxifene (Evista) became available in June 2002, and teriparatide (Forteo), in December 2005 in our hospital. Patients received follow-up during the latter part of this study period, and most patients in the preventive group may have received better medical treatment for osteoporosis. The better control of osteoporosis was demonstrated by the absence of any new incidence of nonadjacent fracture 3 years after vertebroplasty in both the preventive and nonpreventive groups.

However, the improvements in antiosteoporosis medication have not made much difference in the incidence of PVNO adjacent fractures, so PrVP remains necessary for the following reasons: 1) Most patients seek interventional treatment because of VCF with osteoporosis that was not adequately treated before the vertebroplasty, 2) medications for osteoporosis take months to become effective in the prevention of new VCF, and 3) most adjacent fractures developed within several months after vertebroplasty- $62 \%$ of adjacent fractures occurred within 6 months after vertebroplasty in the nonpreventive group. Therefore, PrVP is of value in reducing the incidence of PVNO adjacent VCFs, and its effect was evident within half a year after vertebroplasty by the $21 \%$ reduction in the incidence of any adjacent fracture in this study.

\section{Conclusions}

PVNO VCF in the adjacent vertebral body is a frequent complication after percutaneous vertebroplasty; it occurred in $39 \%$ of our patients in the nonpreventive group and in $26 \%$ of initial vertebrae adjacent to the first TVP levels in the nonpreventive group. In the PrVP group, only 3\% of patients and 2\% of all adjacent vertebrae developed PVNO VCF. Adjacent fracture was not found in vertebrae next to the initial PrVP level and was found in $1(2 \%)$ adjacent vertebra next to the PrVP level, among all PrVP procedures. None of the vertebral bodies that received vertebroplasty for unhealed fracture (TVP) or for prevention (PrVP) developed PVNO VCF. Approximately $33 \%$ of patients in the nonpreventive group underwent repeat vertebroplasty procedures, and most were caused by adjacent fractures. In comparison, only $3 \%$ of patients in the preventive group underwent repeat vertebroplasty procedures. Therefore, our strategy of PrVP did work in the prevention of propagation of vertebral fracture by lowering the incidence of adjacent fracture and reducing the necessity of multiple repeat vertebroplasty procedures.

\section{Acknowledgments}

We are grateful to Chiao-Hsin Chen for patient follow-up and data management and Pui-Ching Lee for statistical analysis.

\section{References}

1. Trout AT, Kallmes DF. Does vertebroplasty cause incident vertebral fractures? A review of available data. AJNR Am J Neuroradiol 2006;27:1397-403

2. Lindsay R, Silverman SL, Cooper C, et al. Risk of new vertebral fractures in the year following a fracture. JAMA 2001;285:320-23

3. Kobayashi N, Numaguchi Y, Fuwa S, et al. Preventive vertebroplasty: cement injection into non-fractured vertebral bodies during percutaneous vertebroplasty. Acad Radiol 2009;16:136-43

4. Komemushi A, Tanigawa N, Kariya S, et al. Percutaneous vertebroplasty for osteoporotic compression fracture: multivariate study of predictors of new vertebral body fracture. Cardiovasc Intervent Radiol 2006;29:580-85

5. Vooemolen MH, Lohle PN, Juttmann JR, et al. The risk of new osteoporotic vertebral compression fracture in the year after percutaneous vertebroplasty. $J$ Vasc Interv Radiol 2006;17:71-76

6. Chang CY, Teng MM, Wei CJ, et al. Percutaneous vertebroplasty for patients with osteoporosis: a one-year follow-up. Acta Radiol 2006;47:568-73

7. Trout AT, Kallmes DF, Layton KF, et al. Vertebral endplate fractures: an indicator of the abnormal forces generated in the spine after vertebroplasty. J Bone Miner Res 2006;21:1797-802

8. Oakland RJ, Furtado NR, Wilcox RK, et al. Preliminary biomechanical evaluation of preventive vertebral reinforcement adjacent to vertebroplasty under cyclic loading. Spine J 2009;9:174-81

9. Lin EP, Ekholm S, Hiwatashi A, et al. Vertebroplasty: cement leakage into the 
disc increases the risk of new fracture of adjacent vertebral body. AJNR Am J Neuroradiol 2004;25:175-80

10. Chiang $\mathrm{CK}$, Wang $\mathrm{YH}$, Yang $\mathrm{CY}$, et al. Preventive vertebroplasty may reduce the risk of adjacent intact vertebrae from fatigue injury. Spine 2009;34:356-64

11. Baround G, Vant C, Wilcox R. Long-term effects of vertebroplasty: adjacent vertebral fractures. J Long Term Eff Med Implants 2006;16:265-80

12. Sun YC, Teng MM, Yuan WS, et al. Risk of post-vertebroplasty fracture in adjacent vertebral bodies appears correlated with the morphologic extent of bone cement. J Chin Med Assoc 2011;74:357-62. Epub 2011 Jul 23

13. Rohlmann A, Zander T, Bergmann G. Spinal loads after osteoporotic vertebral fractures treated by vertebroplasty or kyphoplasty. Eur Spine J 2006; 15:1255-64

14. Becker S, Garoscio M, Meissner J, et al. Is there an indication for preventive balloon kyphoplasty? Clin Orthop 2007;458:83-89 